\title{
A 33 hour period for the Wolf-Rayet/black hole X-ray binary candidate NGC $300 \mathrm{X}-1$
}

\author{
S. Carpano ${ }^{1}$, A. M. T. Pollock ${ }^{1}$, A. Prestwich ${ }^{2}$, P. Crowther ${ }^{3}$, J. Wilms ${ }^{4}$, L. Yungelson ${ }^{5}$, and M. Ehle ${ }^{1}$ \\ 1 XMM-Newton Science Operations Centre, ESAC, ESA, PO Box 50727, 28080 Madrid, Spain \\ e-mail: scarpano@sciops.esa.int \\ 2 Harvard-Smithsonian Center for Astrophysics, Cambridge, MA 02138, USA \\ 3 Department of Physics \& Astronomy, University of Sheffield, Hicks Building, Hounsfield Rd, Sheffield S3 7RH, UK \\ 4 Dr. Remeis-Observatory, Astronomisches Institut der FAU Erlangen-Nürnberg, Sternwartstr. 7, 96049 Bamberg, Germany \\ 5 Institute of Astronomy of the Russian Academy of Sciences, 48 Pyatnitskaya Str., 119017 Moscow, Russia
}

Received 27 February 2007 / Accepted 10 March 2007

\section{ABSTRACT}

\begin{abstract}
Context. NGC $300 \mathrm{X}-1$ is the second extragalactic candidate, after IC $10 \mathrm{X}-1$, in the rare class of Wolf-Rayet/compact object X-ray binary systems exemplified in the Galaxy by Cyg X-3. From a theoretical point of view, accretion onto a black hole in a detached system is possible for large orbital periods only if the mass of the relativistic object is high or the velocity of the accreted wind is low. Aims. We analysed a 2 week SWIFT XRT light curve of NGC 300 X-1 and searched for periodicities.

Methods. Period searches were made using Lomb-Scargle periodogram analysis. We evaluated the confidence level using Monte Carlo simulations.

Results. A period of $32.8 \pm 0.4 \mathrm{~h}$ ( $3 \sigma$ error) was found for NGC $300 \mathrm{X}-1$ with a confidence level $>99 \%$. Furthermore, we confirm the high irregular variability during the high flux level, as already observed in the XMM-Newton observations of the source. A folded $X M M$-Newton light curve is shown, with a profile that is in agreement with SWIFT. The mean absorbed X-ray luminosity in the SWIFT observations was $1.5 \times 10^{38} \mathrm{erg} \mathrm{s}^{-1}$, close to the value derived from the XMM-Newton data.

Conclusions. While Cyg X-3 has a short period of $4.8 \mathrm{~h}$, the period of NGC $300 \mathrm{X}-1$ is very close to that of IC $10 \mathrm{X}-1(34.8 \pm 0.9 \mathrm{~h})$. These are likely orbital periods. Possibility of formation of accretion disk for such high orbital periods strongly depends on the terminal velocity of the Wolf-Rayet star wind and black-hole mass. While low masses are possible for wind velocities $\lesssim 1000 \mathrm{~km} \mathrm{~s}^{-1}$, these increase to several tens of solar masses for velocities $>1600 \mathrm{~km} \mathrm{~s}^{-1}$ and no accretion disk may form for terminal velocities larger than $1900 \mathrm{~km} \mathrm{~s}^{-1}$.
\end{abstract}

Key words. X-rays: individuals: NGC 300 X-1 - X-rays: binaries - stars: Wolf-Rayet

\section{Introduction}

Wolf-Rayet/black hole binaries are believed to be stars in the evolutionary stage following high-mass X-ray binaries. The existence of helium-star/compact object X-ray binaries was suggested independently by van den Heuvel \& de Loore (1973) to explain the nature of the galactic source Cyg X-3, and by Tutukov \& Yungelson (1973) based on results of evolutionary computations. To appear as an X-ray source, an accretion disk must form and hence the velocity of the Wolf-Rayet (WR) star wind must be slow enough for the material around the compact object to be accreted. According to Illarionov \& Sunyaev (1975), a black hole appears as a strong X-ray source in a detached binary system only when the orbital period, $P_{\text {orb }}$ :

$P_{\text {orb }} \lesssim 4.8 \frac{M_{\mathrm{BH}}}{v_{1000}^{4} \delta^{2}}(\mathrm{~h})$

where $M_{\mathrm{BH}}$ is the black hole mass is solar units, $v_{1000}$ is the velocity of the accreted wind in units of $1000 \mathrm{~km} \mathrm{~s}^{-1}$ and $\delta$ is a dimensionless parameter of order unity. It has been shown by Ergma \& Yungelson (1998) and Lommen et al. (2005) that these periods for solar metallicity stars cannot be larger than several tens of hours.
So far, Cyg X-3 is the only valid candidate in our galaxy for a Wolf-Rayet/compact object X-ray binary system. Its X-ray luminosity is high, $L_{\mathrm{X}} \sim 10^{38} \mathrm{erg} \mathrm{s}^{-1}$. The companion star was identified as a WR star by van Kerkwijk et al. (1992) and then designated as WR 145a in the 7th catalogue of galactic WolfRayet stars (van der Hucht 2001). Its orbital period is very short, $4.8 \mathrm{~h}$ (Parsignault et al. 1972).

IC $10 \mathrm{X}-1\left(L_{\mathrm{X}} \sim 1.2 \times 10^{38} \mathrm{erg} \mathrm{s}^{-1}\right)$, in the starburst galaxy IC 10 located at $0.8 \mathrm{Mpc}$, was the first extragalactic candidate for this class of objects (Bauer \& Brandt 2004; Wang et al. 2005). A period of $34.8 \pm 0.9 \mathrm{~h}$ has been observed recently thanks to SWIFT observations (A. Prestwich et al., ATel \#955, paper in preparation). We report in this Letter the discovery of a very similar but slightly shorter period of $32.8 \mathrm{~h}$ for NGC $300 \mathrm{X}-1$, which is the second extragalactic Wolf-Rayet/compact object Xray binary candidate (Carpano et al. 2007).

NGC $300 \mathrm{X}-1$ is the brightest X-ray point source in the dwarf spiral galaxy NGC 300 at a distance of $\sim 1.88 \mathrm{Mpc}$ (Gieren et al. 2005). The galaxy is almost face-on and has a low Galactic column density of $N_{\mathrm{H}}=3.6 \times 10^{20} \mathrm{~cm}^{-2}$ (Dickey \& Lockman 1990). Study of its X-ray population has been done by Read \& Pietsch (2001) using ROSAT and by Carpano et al. (2005) using XMMNewton. Based on the existing four XMM-Newton observations, it has been shown in Carpano et al. (2007) that the position of the 
$\mathrm{X}$-ray source $\left(\alpha_{\mathrm{J} 2000}=00^{\mathrm{h}} 55^{\mathrm{m}} 10^{\mathrm{s}} .00, \delta_{\mathrm{J} 2000}=-37^{\circ} 42^{\prime} 12^{\prime \prime} .^{\prime} 06\right)$ coincides with a WR candidate, WR 41 (Schild et al. 2003), within 0 !' $11 \pm 0$ '. 45 . WR 41 has now been spectroscopically confirmed as an early-type WN star (Crowther et al., in preparation).

The four XMM-Newton light curves, lasting $\sim 10 \mathrm{~h}$ each, showed irregular variability, and during one observation, the flux increased by about a factor of ten in $10 \mathrm{~h}$. No period between $5 \mathrm{~s}$ and $30 \mathrm{ks}(8.3 \mathrm{~h})$ was found in the data. The mean observed (absorbed) luminosity in the $0.2-10 \mathrm{keV}$ band was $\sim 2 \times 10^{38} \mathrm{erg} \mathrm{s}^{-1}$. The unabsorbed X-ray luminosity reached $L_{0.2-10 \mathrm{keV}} \sim 1 \times 10^{39} \mathrm{erg} \mathrm{s}^{-1}$ suggesting the presence of a black hole, altough beamed emission from a neutron star cannot be excluded. The spectrum could be modelled by a power-law with $\Gamma \sim 2.45$ with additional relatively weak emission, notably around $0.95 \mathrm{keV}$.

In this Letter, we report the discovery of a $32.8 \mathrm{~h}$ period for NGC $300 \mathrm{X}-1$. The remainder of the Letter is organised as follows. Section 2 briefly describes the SWIFT observations and data reduction. In Sect. 3, we report analysis of the SWIFT XRT light curve and search for periodicities using a Lomb-Scargle periodogram analysis. A folded XMM-Newton light curve is shown in Sect. 4, while a discussion of our results is given in Sect. 5.

\section{Observations and data reduction}

NGC 300 X-1 was observed with the SWIFT Gamma-Ray Burst Explorer (Gehrels et al. 2004) between 2006 December 26 and 2007 January 10, for a total of $83 \mathrm{ks}$. The light curve of NGC 300 X-1 was extracted from the X-ray Telescope, XRT (Burrows et al. 2005) which operates in the $0.2-10 \mathrm{keV}$ energy band. There are 146 XRT observations lasting between 10 and 1477 s. We kept only data from 124 observations lasting more than $100 \mathrm{~s}$.

For the production of the X-ray light curve, we analysed the calibrated and screened PC event files (level 2) provided in the set of data products. Source and background regions were extracted using the FTOOLS ${ }^{1}$ ftselect task. The circular source region was centred on the XMM-Newton source position $\left(\alpha_{\mathrm{J} 2000}=00^{\mathrm{h}} 55^{\mathrm{m}} 10^{\mathrm{s}} .00, \delta_{\mathrm{J} 2000}=-37^{\circ} 42^{\prime} 12^{\prime \prime}\right.$.06 $)$ with a radius of $40^{\prime \prime}$, which is larger than the telescope PSF (18"). A similar sized region was extracted for the background, in a blank region close to NGC $300 \mathrm{X}-1$.

\section{Time analysis of SWIFT light curve}

The SWIFT XRT background-subtracted light curve is shown in Fig. 1. Times are given in hours from the beginning of the observation. We overplotted the best-fit sinusoid function. For clarity, the amplitude has been multiplied by a factor of 1.5 . It is clear that the flux varies in a regular way, with the minima likely to be eclipses of the accreting companion.

We searched for a periodic signal between 5 and $100 \mathrm{~h}$, using a Lomb-Scargle periodogram analysis (Lomb 1976; Scargle 1982). By means of Monte Carlo simulations, we evaluated the confidence level assuming a null hypothesis of white noise. Results are plotted in Fig. 2. The full, dashed and dotted lines represent the $68 \%, 90 \%$ and $99 \%$ confidence level respectively. We found that the $32.84 \mathrm{~h}$ period is significant at a confidence level $>99 \%$. To estimate the error, we fitted a sine function using the IDL task curvefit keeping trial periods fixed. The reduced chi-square, $\chi_{v}^{2}$ with $v=121$, is shown in Fig. 3 . The full, dotted

\footnotetext{
${ }^{1}$ http://heasarc.nasa.gov/lheasoft/ftools
}

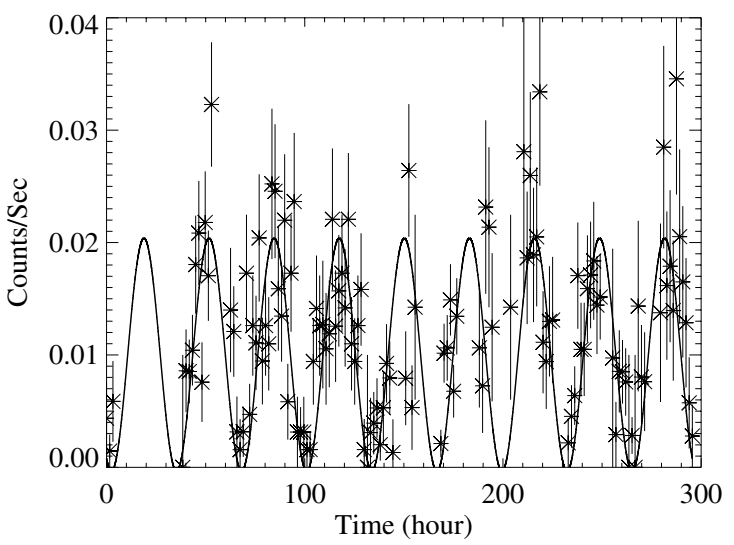

Fig. 1. SWIFT XRT background-substracted light curve of NGC $300 \mathrm{X}-1$. Overplotted is the best-fit sinusoid function.

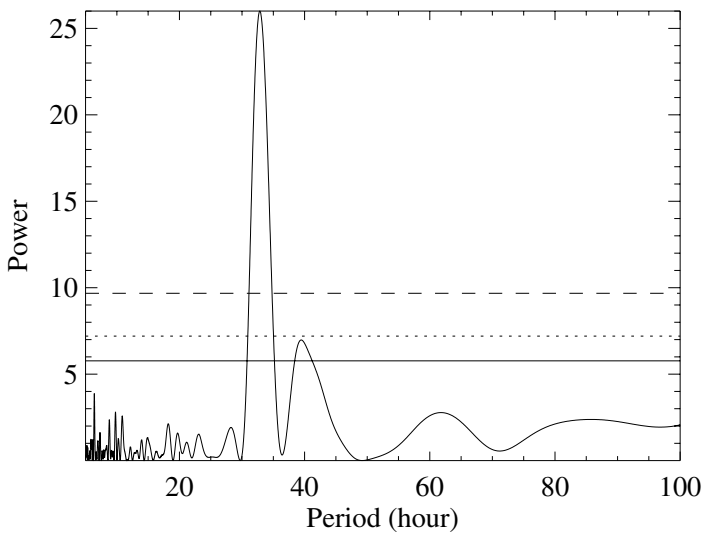

Fig. 2. Search for periodicities for the SWIFT XRT light curve of NGC 300 X-1 using a Lomb-Scargle periodogram analysis. The full, dotted and dashed lines represent the 68\%, 90\% and 99\% confidence level respectively.

and dashed lines represent $\Delta \chi^{2}=1.00,2.71$ and 6.63 respectively. The corresponding 1,2 and $3 \sigma$ period range are [32.6733.00], [32.57-33.12] and [32.42-33.28] respectively. Note that the $\chi_{v}^{2}$ larger than 1 shows that the light curve cannot be described by a pure sinusoid function.

The SWIFT XRT light curve folded at $32.84 \mathrm{~h}$ is shown in Fig. 4. Phase zero is associated to the beginning of the first SWIFT observation. From Figs. 1 and 4, we confirm irregular high variability outside the eclipse as observed in the XMM-Newton data (Carpano et al. 2007).

We estimate the X-ray luminosity by converting the mean count rate, 0.012 count $\mathrm{s}^{-1}$ to flux with WebPIMMS ${ }^{2}$, using the spectral parameters derived by Carpano et al. (2007). The mean absorbed luminosity in the $0.2-10 \mathrm{keV}$ energy band is $1.5 \times$ $10^{38} \mathrm{erg} \mathrm{s}^{-1}$, which is close to that found from the XMM-Newton data, $L_{0.2-10 \mathrm{keV}} \sim 2 \times 10^{38} \mathrm{erg} \mathrm{s}^{-1}$ (Carpano et al. 2007) and close to the ROSAT value, $L_{0.1-2.4 \mathrm{keV}} \sim 2.2 \times 10^{38} \mathrm{erg} \mathrm{s}^{-1}($ Read \& Pietsch 2001).

\footnotetext{
${ }^{2}$ http://heasarc.gsfc.nasa.gov/Tools/w3pimms.html
} 


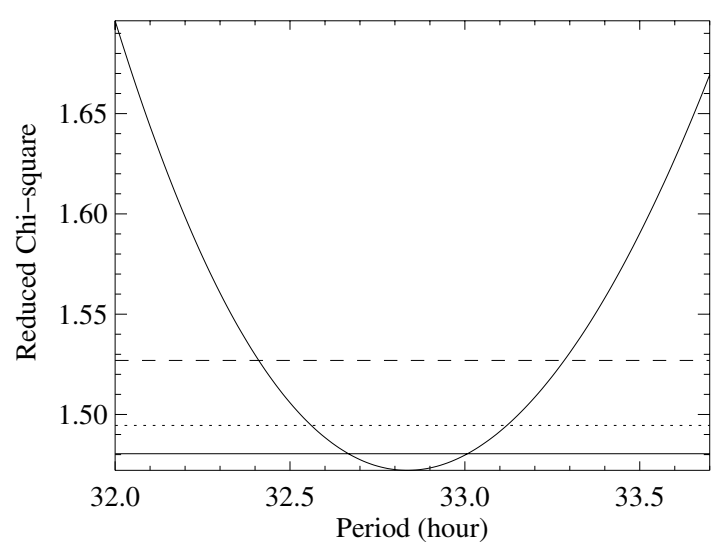

Fig. 3. Period error search fitting sine curve. The full, dotted and dashed lines represent $\Delta \chi^{2}=1.00,2.71$ and 6.63 respectively.

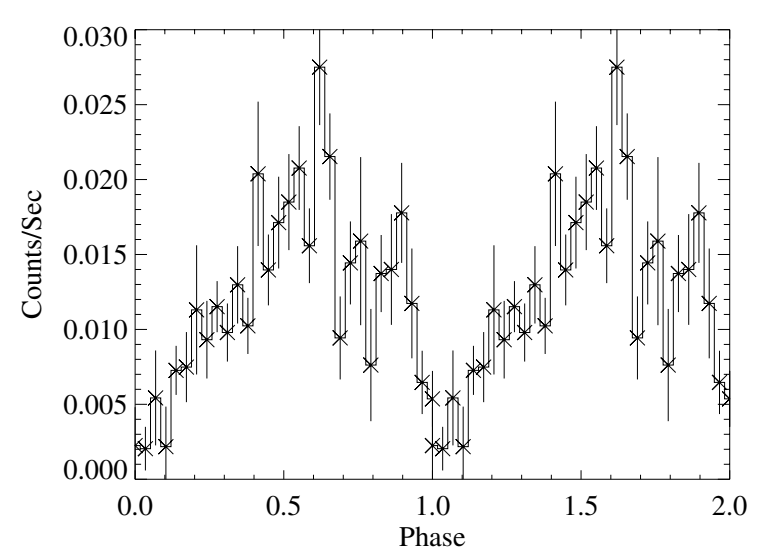

Fig. 4. SWIFT XRT light curve folded at $32.84 \mathrm{~h}$ using 30 bins. Phase zero is associated to the beginning of the first SWIFT observation.

\section{XMM-Newton folded light curve}

We searched for periodicity in the four XMM-Newton data samples observed between 2000 and 2005 (see Carpano et al. (2006) for more details about the observations). Although the unfavourable sampling precludes a rigorous period search, we have used some a priori information we have on the eclipse profile to fold the light curve. From Table 1 and Fig. 2 of Carpano et al. (2007), we can compare the flux and light curve shape of the several observations to the SWIFT profile of Fig. 4. The flux was minimum in the first XMM-Newton observation and began to increase at the end. This could be associated to the eclipse state of the X-ray source. The second XMM-Newton observation, 6 days later, is likely associated with an eclipse egress. In the third observation the flux is lower than in the second and fourth observations, and shows a small decrease trend: this data set could be associated with the beginning of the eclipse ingress. And in the fourth XMM-Newton observation, the flux is high and likely outside the eclipse. We used this information to constrain the phase of each beginning of data set, in the folded light curve. Only few values of the period around $33 \mathrm{~h}$, but larger than $32.8 \mathrm{~h}$, are possible to provide a reasonable profile.

Figure 5 shows the XMM-Newton EPIC MOS light curve folded at a period of $33.066 \mathrm{~h}$, within the $2 \sigma$ error of the SWIFT period. Phase zero is associated with the start of the

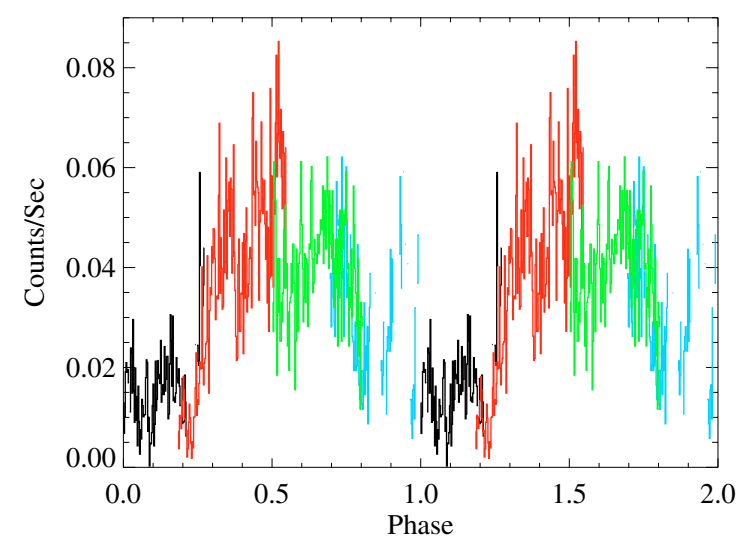

Fig. 5. MOS XMM-Newton light curve folded at a period of $33.066 \mathrm{~h}$ using time bins of $300 \mathrm{~s}$ (Carpano et al. 2007). First observation is in black, second in red, third in blue and fourth in green. Phase zero is associated with the start of the XMM-Newton observations and is by chance during eclipse as for the SWIFT data.

XMM-Newton observations and is by chance during eclipse as for the SWIFT data. Comparing Figs. 4 and 5, it seems that both curves have a small dip around phase $0.6-0.8$. Further observations of the source are clearly necessary to confirm this and other features of the periodic light curve.

\section{Discussion}

In the evolutionary scenario for WR/compact object X-ray binaries that has been suggested to explain the short orbital period observed in Cyg X-3, the immediate precursor of the system is a neutron star or black hole orbiting an OB star. When the latter leaves the main sequence, matter is transferred due to Roche lobe overflow and a common envelope forms. Due to friction, the distance between the early-type star core and the compact object decreases. A merger is avoided if the binding energy of the hydrogen envelope is lower than the energy released by spiral-in, leading to a short-period binary system consisting of a WR star and a compact object. Formation of an accretion disk around a black hole from the strong wind of the helium star is then possible if the orbital period satisfies Eq. (1).

We now derive possible values for the masses of the black hole and the Wolf-Rayet star that allow the formation of an accretion disk for an orbital period of $32.8 \mathrm{~h}$. Kepler's third law gives:

$a=0.506 P_{\text {orb }}^{2 / 3}\left(M_{\mathrm{BH}}+M_{\mathrm{WR}}\right)^{1 / 3}\left(R_{\odot}\right)$

where, for a circular orbit, $a$ is the binary separation, $P_{\text {orb }}$ the orbital period in hours, and $M_{\mathrm{BH}}$ and $M_{\mathrm{WR}}$ the component masses in solar units. The velocity of the Wolf-Rayet wind, $v_{\mathrm{WR}}$ at $a$ can be approximated by a $\beta$-law (Lamers \& Cassinelli 1999):

$v_{\mathrm{WR}}(a)=v_{0}+\left(v_{\infty}-v_{0}\right)\left(1-\frac{R_{\mathrm{WR}}}{a}\right)^{\beta}$

where $R_{\mathrm{WR}}$ is the Wolf-Rayet star radius (in $R_{\odot}$ ), $v_{\infty}$ the terminal velocity, $v_{0}$ the initial velocity $\left(\sim 0.01 v_{\infty}\right)$ and the $\beta$ parameter describes the steepness of the law. Note that $\beta=1$ is the preferred value for Wolf-Rayet winds (Gräfener \& Hamann 2005). For the radii of Wolf-Rayet stars, we can use the relation given by Schaerer \& Maeder (1992):

$\log \left(R_{\mathrm{WR}}\right)=-0.6629+0.5840 \log \left(M_{\mathrm{WR}}\right)$. 

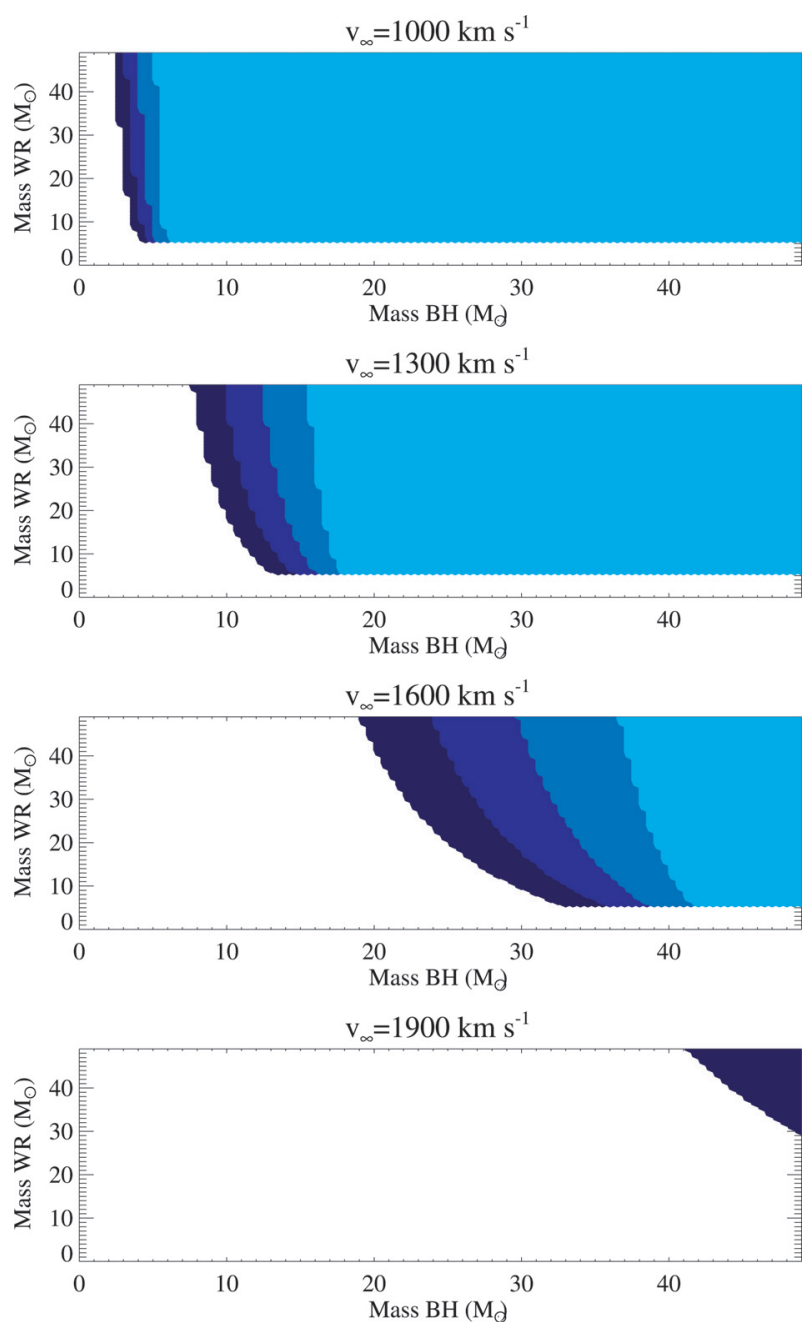

Fig. 6. Allowed WR and BH masses, in solar units, that satisfy the condition for the formation of an accretion disk for different values of the terminal velocity of the wind. Different values of the wind velocity $\beta$ parameter $(2.0,1.5,1.0$ and 0.5$)$ are represented by dark to light blue. For each value of $\beta$, the allowed region of $M_{\mathrm{WR}}-M_{\mathrm{BH}}$ parameter space extents all the way up to the right.

Given that orbital period of the system is known, Eqs. (1)-(4) define combinations of the component masses for which an accretion disk may form. The plots are shown in Fig. 6 for different values of the $\beta$ parameter and the terminal velocity. We restrict to $M_{\mathrm{WR}}>7 M_{\odot}$ and $M_{\mathrm{BH}}>3 M_{\odot}$, which are standard lower limit for the WR and BH masses respectively.

Looking at these graphs we can note that, for a terminal velocity around $1000 \mathrm{~km} \mathrm{~s}^{-1}$, whatever the mass of the Wolf-Rayet star and the value of the $\beta$ parameter, the lower limit for the black hole mass is below $7 M_{\odot}$. With higher values for the terminal velocity, this lower limit increases significantly and becomes more and more dependent on the $\beta$ parameter. For velocities of $1600 \mathrm{~km} \mathrm{~s}^{-1}$, the mass must be at least several tens of solar masses, while no accretion disk may form for terminal velocities significantly higher than $1900, \mathrm{~km} \mathrm{~s}^{-1}$. From the optical spectrum of WR 41, the terminal velocity of the wind is about $1250 \mathrm{~km} \mathrm{~s}^{-1}$ and the mass of the Wolf-Rayet star is estimated between $18 M_{\odot}$ and $40 M_{\odot}$ (Crowther et al., in preparation). This leads to a black hole mass for NGC $300 \mathrm{X}-1$ larger than $13 M_{\odot}$ for $\beta=1$.

Similar arguments apply for IC $10 \mathrm{X}-1$ : the mass of the WolfRayet star was derived to be $35 M_{\odot}$ and its terminal wind velocity $1750 \mathrm{~km} \mathrm{~s}^{-1}$ (Clark \& Crowther 2004). This means that the mass of the black hole companion must be at least of $\sim 35 M_{\odot}$, for an orbital period of $34.8 \mathrm{~h}$.

To conclude, it seems a surprise that orbital periods found in both extragalactic WR/compact object X-ray binary candidates IC $10 \mathrm{X}-1$ and NGC $300 \mathrm{X}-1$ are so similar. Furthermore, their difference to the short period of Cyg X-3 may suggest different paths of evolution.

Acknowledgements. This paper is based on observations obtained with the SWIFT gamma-ray burst mission and observations from XMM-Newton, an ESA science mission with instruments and contributions directly finded by ESA Member States and NASA. L.Y. is supported by the Russian Academy of Sciences grant "Origin and evolution of stars and galaxies" and NSF grant No. PHY99-07949. We warmly thank Neil Gehrels and Dave Burrows for approving the SWIFT observing time.

\section{References}

Bauer, F. E., \& Brandt, W. N. 2004, ApJ, 601, L67

Burrows, D. N., Hill, J. E., Nousek, J. A., et al. 2005, Space Sci. Rev., 120, 165 Carpano, S., Wilms, J., Schirmer, M., \& Kendziorra, E. 2005, A\&A, 443, 103

Carpano, S., Wilms, J., Schirmer, M., \& Kendziorra, E. 2006, A\&A, 458, 747

Carpano, S., Pollock, A. M. T., Wilms, J., Ehle, M., \& Schirmer, M. 2007, A\&A, 461, L9

Clark, J. S., \& Crowther, P. A. 2004, A\&A, 414, L45

Dickey, J. M., \& Lockman, F. J. 1990, ARA\&A, 28, 215

Ergma, E., \& Yungelson, L. R. 1998, A\&A, 333, 151

Gehrels, N., Chincarini, G., Giommi, P., et al. 2004, ApJ, 611, 1005

Gieren, W., Pietrzyński, G., Soszyński, I., et al. 2005, ApJ, 628, 695

Gräfener, G., \& Hamann, W.-R. 2005, A\&A, 432, 633

Illarionov, A. F., \& Sunyaev, R. A., A\&A, 39, 185

Lamers, H. J. G. L. M., \& Cassinelli, J. P. 1999, Introduction to Stellar Winds (Cambridge, UK: Cambridge University Press)

Lomb, N. R. 1976, Ap\&SS, 39, 447

Lommen, D., Yungelson, L., van den Heuvel, E., Nelemans, G., \& Portegies Zwart, S. 2005, A\&A, 443, 231

Parsignault, D. R., Gursky, H., Kellogg, E. M., et al. 1972, Nature, 239, 123

Read, A. M., \& Pietsch, W. 2001, A\&A, 373, 473

Scargle, J. D. 1982, ApJ, 263, 835

Schaerer, D., \& Maeder, A. 1992, A\&A, 263, 129

Schild, H., Crowther, P. A., Abbott, J. B., \& Schmutz, W. 2003, A\&A, 397, 859

Tutukov, A., \& Yungelson, L. 1973, Nauchnye Informatsii, 27, 70

van den Heuvel, E. P. J., \& de Loore, C. 1973, A\&A, 25, 387

van der Hucht, K. A. 2001, New Astron. Rev., 45, 135

van Kerkwijk, M. H., Charles, P. A., Geballe, T. R., et al. 1992, Nature, 355, 703

Wang, Q. D., Whitaker, K. E., \& Williams, R. 2005, MNRAS, 362, 1065 